\title{
The Liar Hypodox: A Truth-Teller's Guide to Defusing Proofs of the Liar Paradox
}

\author{
Peter Eldridge-Smith \\ Philosophy, Research School of the Social Sciences, Australian National University, Canberra, Australia \\ Email: peter.eldridge-smith@anu.edu.au, peter.eldridgesmith@gmail.com
}

How to cite this paper: Eldridge-Smith, P. (2019). The Liar Hypodox: A Truth-Teller's Guide to Defusing Proofs of the Liar Paradox. Open Journal of Philosophy, 9, 152-171. https://doi.org/10.4236/ojpp.2019.92011

Received: February 21, 2019

Accepted: May 5, 2019

Published: May 8, 2019

Copyright $\odot 2019$ by author(s) and Scientific Research Publishing Inc. This work is licensed under the Creative Commons Attribution International License (CC BY 4.0).

http://creativecommons.org/licenses/by/4.0/

\begin{abstract}
It seems that the Truth-teller is either true or false, but there is no accepted principle determining which it is. From this point of view, the Truth-teller is a hypodox. A hypodox is a conundrum like a paradox, but consistent. Sometimes, accepting an additional principle will convert a hypodox into a paradox. Conversely, in some cases, retracting or restricting a principle will convert a paradox to a hypodox. This last point suggests a new method of avoiding inconsistency. This article provides a significant example. The Liar paradox can be defused to a hypodox by relatively minimally restricting three principles: the T-schema, substitution of identicals and universal instantiation. These restrictions are not arbitrary. For each, I identify the source of a contradiction given some presumptions. Then I propose each restriction as a reasonable way to deal with that source of contradiction.
\end{abstract}

\section{Keywords}

Liar paradox, Hypodox, Truth, T-Schema, Substitution of Identicals

\section{Introduction}

Compared to the Liar paradox, the Truth-teller is a squib. Proofs of the Liar paradox seemingly soundly entail a contradiction, from which anything (and everything) follows in classical logic by Explosion (ex contradictione quodlibet). In contrast, the Truth-teller's proofs-it does have some-seem to have too few logical consequences. These are short seemingly sound proofs, given certain logical principles, that the Truth-teller sentence (statement, etc.) is either true or not true; but there seems no accepted principled way to conclude which, and no contingency will resolve the matter. From this point of view, Truth-tellers seem underdetermined and paradigm examples of hypodoxes (Eldridge-Smith 2007, 2012, 2015: Appendix). Sometimes, accepting an additional principle will con- 
vert a hypodox into a paradox (Eldridge-Smith 2012). Conversely, in some cases, retracting or restricting a principle will convert a paradox to a hypodox. This last point suggests a new method of (at least partially) resolving some paradoxes. This article provides a significant example. The Liar paradox can be defused to a hypodox by relatively minimally restricting three principles: the T-schema, substitution of identicals and universal instantiation. These restrictions are not arbitrary. I identify several sources of a contradiction given some presumptions. In relation to each of these, each restriction is a reasonable way to deal with that source of contradiction. The restrictions aim at invalidating just those pathological inferences that otherwise result in a Liar contradiction by invalidating just its sources of contradiction. A case for surgery over living with a pathology is not required, because one cannot actually live with the pathology of the Liar paradox on pain of Explosion. Some principles must be restricted; that is, as Quine points out, the Liar forces us to revise some trusted way of reasoning (Quine 1976). What I propose excises minimally from otherwise trusted patterns of reasoning.

Let me provide some general background. The Liar paradox concerns any statement that says of itself that it is false (or not true) and an otherwise seemingly acceptable argument about that statement to a contradiction (cf. Sainsbury, 2009: p. 1, and pp. 127-129; and Cook, 2013: p.14). Here is an example:

If I say that I am lying [and nothing else], am I telling the truth? If I am, I am lying and so uttering a falsehood; but if I am not telling the truth, I am lying, and so I am telling the truth. So my utterance is both true and false.

(Clark, 2012: p. 118)

This example uses "lying" simply as a synonym for not telling the truth. This could be challenged, but the paradox is generally considered as an obstacle for a theory of truth. The seminal work towards such a theory is Tarski (1935). Among other innovations, Tarski articulated the trusted ways we reason about truth by means of a schema that I will present in the next section.

Liar sentences can generally be paired with Truth-teller sentences. For example, the self-referential sentence "This sentence is false" can be paired with the self-referential sentence "This sentence is true". The latter is an example of a Truth-teller. As a grammatical sentence, one can argue that this Truth-teller sentence is either true or not true; but we seem to lack any way of reasoning to a conclusion about which it is. Unlike the Liar paradox, the Truth-teller hypodox seems consistent but its truth-value seems underdetermined. This is typical of hypodoxes (see Eldridge-Smith 2007, esp. endnote 3; and 2012), and I have conjectured that any hypodox can be paired with at least one dual paradox (Eldridge-Smith 2018: Appendix). But it is just the Truth-teller that concerns us here. Reasoning about the Truth-teller will be our guide to correcting some of our reasoning about truth in two ways that otherwise lead to Liar contradictions. These are: first, substitution of identicals, the trusted way of reasoning that if two terms refer to the same object, the terms may be substituted for each other in 
any sentence while preserving the truth-value of that sentence; and secondly, universal instantiation, the trusted way of reasoning from a universal statement about, for example, all film stars to a particular statement about Nicole Kidman.

Apart from Tarski's work, many modern approaches to the Liar paradox advocate modifications to sentential logic. For an overview of these approaches see Cook (2012: chapters 2 and 3). There are also some sophisticated technical approaches working at the "substructural" level of argument by, for example, placing restrictions on the transitivity of inferences. For a good example of this approach, see Ripley (2013). In contrast, my approach is that the Liar has a complex cause, arising from several issues with predicate logic when it is extended with a truth predicate. The way in which I extend the logic with a truth predicate is similar, in some respects, to some axiomatic approaches. For a canonical account of axiomatic truth theory, see Halbach (2014). To the best of my knowledge, I am the first person to argue for a failure of substitution of identicals to avoid the Liar contradiction without also requiring consequent changes in sentential logic (SL). The first suggestion that substitution of identicals should be restricted to avoid the Liar paradox was made by Skyrms (1970). But Skyrms' argument presumes that the Liar is neither true nor false; something that I do not presume. Indeed, I aim to defuse the Liar sentence to a hypodox, a statement which is either true or not true, although it is underdetermined which it is.

My method is to identify three sources of contradiction in Liar arguments using a natural deduction system extended with inference rules for the truth predicate. In the first two cases, I argue that a single inference fails to preserve truth. That is, I show that if the premise of the inference is true, the conclusion is false; therefore, the inference is invalid. In the third case, two inferences are involved; so my argument is a bit more complex. In each case, I make my presumptions explicit in the text. I then recommend minimal restrictions to these rules of inference designed in a reasonable way to avoid these sources of contradiction.

The structure of the article proceeds from the simplest case of such a source of contradiction and its remediation in Section 2. In Section 3, I then explain how comparison between Truth-teller inferences and corresponding inferences used in the Liar paradox demonstrates that substitution of identicals fails in these cases. I explain and demonstrate how this can be remediated with a minimal restriction on the rule for substitution of identicals in first-order predicate logic. This is sufficient to reduce variations of the Liar that do not use quantification to hypodoxes, which I show in Sections 4 and 5. Then in section 6, I address a third source of contradiction in Liar arguments involving quantification. I show how that source can be remediated so that quantified Liars, like the Epimenides paradox, are defused to hypodoxes. In Section 7, I make a very qualified claim about the consistency of a system implementing this approach. Finally, I summarize my argument with respect to my major results in the Conclusion.

\section{Tarski's T-Schema Is Informal and Fallacious}

Tarski's T-schema (1944: p. 344) is semantic, and not purely formal. 
(T-schema) $\sigma$ is true if, and only if, $\psi$

where " $\psi$ " is replaced by any sentence and " $\sigma$ " by a name of that sentence.

To see the informality of this schema, consider the following argument that it semantically validates:

(1) The favourite sentence of the author is identical with "The favourite sentence of the author is not true". Premise

(3) The favourite sentence of the author is true if, and only if, the favourite sentence of the author is not true. (1) T-schema

By (1), "The favourite sentence of the author" is a name for the sentence "The favourite sentence of the author is not true", which satisfies the informal condition on the T-schema. To elaborate, (1) is an identity premise. On the standard interpretation of an identity premise, the expressions on either side of "is identical with" in (1) are co-referential expressions. Thus, the expression on the left-hand side is a name for the sentence enquoted on the right-hand-side of (1), and the condition on the T-schema is satisfied (cf. Tarski 1944: p. 347). Therefore, given (1), (3) follows directly by the T-schema.

Why then does (3) seem false? My theory presumes:

(a) (3) is false.

This is the simplest explanation as to why (3) seems false. Yet, in the context of the current debate, $(\alpha)$ asserts that either (3) is a dialetheia (both true and false) or (3) is false and not true. Still, ( $\alpha$ ) does deny that (3) has a truth-value gap. ${ }^{1}$

I also presume that:

( $\beta$ ) (1) makes grammatically acceptable use of names for a sentence.

$(\gamma)$ The sentence mentioned in (1) is grammatically acceptable.

(d) (1) is true.

$(\varepsilon)$ The use of "is true" in (3) is grammatically acceptable.

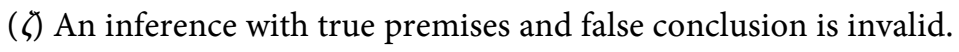

$(\eta)(\zeta)$ is not a dialetheia.

Actually, (1) is contingently true. (I personally do not think that (1) is dialetheic, but my argument does not depend on this.) Using quotes is a grammatical and canonical way of referring to a sentence. "The favourite sentence of the author" is a grammatical definite description that also refers to the sentence enquoted in (1). Besides, syntactic functions can generate a sentence analogous to (1), for which see Quine (1995) or Section 3.3 below. Moreover, decrying (1) or (3) as ungrammatical without an independent reason to do so would be ad hoc. So, I think $(\beta)$ through $(\varepsilon)$ are reasonable (i.e. not ad hoc).

\footnotetext{
${ }^{1}$ I am not alone in thinking biconditionals like (3) are false. Tarski (1944) holds that biconditionals like (3) are contradictory, even though he ultimately eschews natural language as incoherent. This is actually a strong point of divergence between Tarski and three-valued theories. Both "glut" and "gap" theories using the strong Kleene truth tables hold that biconditionals like (3) are true! See Priest (1979) for a dialetheist account, and Kripke (1975) for a truth-value gap account. Since, in those theories, both components on either side of the biconditional in (3) take the third value using the strong Kleene tables, they are equivalent and the biconditional is true (Cook 2013). So, according to those theories (3) is true, whether the third value is interpreted as a glut (both true and false) or a gap (not true and not false).
} 
$(\zeta)$ is entailed by the semantic definition of validity, some sentential logic and the scholastic principle that what is actual is possible; so I think $(\zeta)$ is reasonable. Personally, I think $(\zeta)$ excludes an argument with true premises and a false conclusion from being valid even in cases where the premises or conclusion are dialetheic; but in deference to anyone who thinks otherwise I have added $(\eta)$ as a presumption. Thus, in a theory that grants $(\alpha)$ through to $(\eta)$, the T-schema is fallacious. It seemed like a good principle of truth, but it has counterexamples such as the above argument from a true premise (1) to false conclusion (3).

\section{Rules of Inference That Suggest How to Formalize and Restrict the T-Schema}

Formalizing the inference from (1) to (3) would help to further investigate this fallacy. So, it is reasonable to formalize the condition on the T-schema, if possible. The T-schema is informal because one must be cognizant of the semantic information that " $\sigma$ " is a name of the sentence $\psi$ in order to use that schema. If instead, one restricts the schema to using canonical names of sentences in the language, then one does not need semantic information. This is because it can be mechanically determined whether some term is a canonical name for an expression, and conversely, a canonical name can be mechanically derived from an expression. If instead of the informal T-schema, one uses a schema restricted to canonical names, then (3) does not follow directly from (1). This idea is progressed in this section.

Let us consider a "natural" logical system that satisfies $(\alpha)$ through $(\eta)$. For each logical constant there is a reasonable introduction rule and an elimination rule. For quantifiers, identity and connectives the introduction and elimination rules are chosen such that $(\alpha)$ is the case. Add to our system a means of canonically naming its own sentences. It may have non-canonical names for some sentences as well. Furthermore, add a logical constant "T" as a truth predicate. One way or another, $(\beta)$ through $(\eta)$ are satisfied by the system. Wanted is a rule for introducing truth and a rule for eliminating truth that do not by themselves entail (3), and yet uphold something like the T-schema. Friedman \& Sheard (1987) canvas a wide variety of alternative candidates. The strongest my theory supports in view of the T-schema being fallacious and the ideas motivating this section are the rules below, which are restricted to canonical names.

Truth Introduction (TI): $\psi+\mathrm{T}\langle\psi\rangle$

Truth Elimination (TE): $\mathrm{T}\langle\psi\rangle \vdash \psi$

where " $\psi$ " is any grammatical, closed sentence; the angle brackets expression is replaced by a canonical name of that sentence; and " $\mathrm{T}$ " is the truth predicate, a logical constant that is introduced and eliminated by these rules.

Derived from these with sentential logic, there is a formal schema closely related to the $\mathrm{T}$-schema that restricts introducing and eliminating truth such that each is only valid where it applies to canonical names of sentences. This is the Canonical Truth Schema.

(CT-schema) $\langle\psi\rangle$ is true if and only if $\psi$ 
More succinctly: $\mathrm{T}\langle\psi\rangle \leftrightarrow \psi$

where " $\psi$ " is replaced by any grammatical, closed sentence; the angle brackets expression is replaced by a canonical name of that sentence; and " $\mathrm{T}$ " is the truth predicate.

Let the system use TI and TE. Moreover, let the system be such that each instance of the CT-schema is a logical truth. Since the canonical name of a sentence can be derived mechanically from a given sentence and that sentence can be recovered mechanically from its canonical name, this schema is formal. ${ }^{2}$

If the T-schema had strong intuitive support, then the CT-schema has even stronger intuitive support, simply because the CT-schema only validates a proper subset of the biconditionals that are validated by the T-schema. In particular, (3) is not an instance of the CT-schema even given (1), because (3) applies truth to a non-canonical name on the left-hand-side of the biconditional in (3).

So (3) is not immediately true given the CT-schema and (1); however, it follows by Identity Elimination from (1) and (2), which is an instance of the CT-Schema.

(2) "The favourite sentence of the author is not true" is true if, and only if, the favourite sentence of the author is not true. CT-schema

Thus, given $(\alpha)$ through $(\eta)$, either this instance of Identity Elimination is fallacious, or the CT-schema is fallacious. A further systematic restriction that avoids deriving (3) is motivated in the next section, guided by considerations about the Truth-teller as a hypodox.

\section{Two Rules That Introduce and Eliminate the Truth Predicate Are Too Many}

Some hypodoxes do not need the principles at issue in their related paradoxes (Eldridge-Smith 2012; 2015 Appendix). In particular, the Truth-teller does not need the CT-schema, whereas the proof of a Liar requires some such principle of truth. One significance of this phenomenon is that it exemplifies that there is more than one way of introducing the truth predicate for the Truth-teller, and more than one way of eliminating the truth predicate when predicated of the Truth-teller. This seemingly harmless redundancy with respect to the Truth-teller is correlated with a source of contradiction for the Liar. Consider a Truth-teller, Te, such that $e=\langle\mathrm{T} e\rangle$. (For example, let " $e$ " abbreviate "The second favourite ${ }^{2}$ Cook (2012) brilliantly poses a new problem for taking the T-schema to be a logical truth. This problem does not beset the CT-schema given that canonical names are formal names, the truth predicate is a logical constant and instances of the CT-schema are logical truths. Given these considerations, both the canonical names of expressions and the truth predicate are formal constants, and so they cannot be substituted in the ways that Cook does in his proof that the T-schema is not a logical truth. Cook (2012: p. 238) mentions Jc Beall and Bruno Whittle as having suggested similar ideas for saving a formal T-schema from Cook's new problem. In discussing those ideas, Cook requires some explanation as to why the name for a sentence should be a logical name and why each instance of the CT-schema is a logical truth, and not merely a necessary truth. In our proposal, the names are canonical names, taken to be logical constants in a formal system; the " $\mathrm{T}$ " predicate is a logical constant (as is “=”), and the instances of the CT-schema are derivable just from purely logical rules (TI, TE and SL). 
sentence of the author", then "Te" represents "The second favourite sentence of the author is true".)

On the one hand, $\mathrm{T} e$ and $e=\langle\mathrm{T} e\rangle$ entail $\mathrm{T}\langle\mathrm{T} e\rangle$ by $=\mathrm{E}$; and on the other hand, $\mathrm{T} e$ entails $\mathrm{T}\langle\mathrm{T} e\rangle$ by TI. So $=\mathrm{E}$ has the same effect as TI, that is, $=\mathrm{E}$ and TI overdetermine each other's consequences in this case. Again, for TE, $\mathrm{T}\langle\mathrm{T} e\rangle$ and $e=$ $\langle\mathrm{T} e\rangle$ entail $\mathrm{T} e$ by either $=\mathrm{E}$ and $\mathrm{TE}$, so again $=\mathrm{E}$ has the same effect - the $\mathrm{TE}$ and $=\mathrm{E}$ rules overdetermine how the truth predicate is eliminated in this case. Although overdetermined, the introduction and elimination of truth was consistent; it is redundant in these cases. However, this overdetermination becomes inconsistent in the case of a dual Liar sentence. Paralleling the above entailments, on the one hand, $\sim \mathrm{T} a$ and $a=\langle\sim \mathrm{T} a\rangle$ entail $\sim \mathrm{T}\langle\sim \mathrm{T} a\rangle$ by $=\mathrm{E}$, but on the other $\sim \mathrm{T} a$ entails $\mathrm{T}\langle\sim \mathrm{T} a\rangle$ by TI. Furthermore, $\mathrm{T}\langle\sim \mathrm{T} a\rangle$ and $a=\langle\sim \mathrm{T} a\rangle$ entail Ta by $=\mathrm{E}$, but $\mathrm{T}\langle\sim \mathrm{T}$ a $\rangle$ entails $\sim \mathrm{T} a$ by $\mathrm{TE}$. In these cases, $=\mathrm{E}$ is used to derive expressions that contradict the results of using TI and TE. This source of a Liar inconsistency is avoided if the system is restricted to just one, non-redundant way of introducing and eliminating truth in the case of the Truth-teller that also restricts the correlative inferences made involving the Liar sentence in place of the Truth-teller. In a system with such a restriction, this source of contradiction goes away. Again, to be clear, the contradiction did not arise in cases of the Truth-teller where the use of $=\mathrm{E}$ redundantly duplicates what is derivable using TI or TE, the contradiction arises in the dual Liar cases where the use of $=\mathrm{E}$ entails expressions that contradict those derivable using TI and TE. Our logic is exposed to this contradiction because it has two rules for introducing and eliminating truth in hypodoxical cases that give different results in the paradoxical cases; so, if one of these rules is restricted so as not to overdetermine the results of introducing or eliminating truth in these hypodoxical cases, that same restriction avoids deriving a contradiction in the dual Liar cases.

$\mathrm{TI}$ and TE are meant to be the rules for introducing and eliminating the truth predicate. It is Identity Elimination (=E) that is overdetermining those rules by providing the additional means of introducing and eliminating the truth predicate in combination with canonical names. To avoid overdetermining how the truth predicate can be introduced or eliminated, the Identity Elimination (=E) rule should be restricted from doing so. The effects of this restriction are intended to make use of TI or TE necessary in derivations of the Truth-teller and restrict use of $=\mathrm{E}$ from deriving Liar contradictions.

The above reasoning only seems to occur under an assumption or built into the complexity of a compound formula. For example, when one assumes $\sim \mathrm{Ta}$ and argues to a contradiction. If so, Identity Elimination need only be restricted when used under an assumption or in a compound formula. For present purposes, this is an eighth presumption.

$(\theta)$ The system without a restriction on Identity Elimination is such that inconsistent overdetermination of the results of truth introduction or elimination only occurs under some assumption or using a compound formula. 


\subsection{Restriction of Identity Elimination (=E)}

The following restriction of Identity Elimination aims at avoiding it being used as a rule to introduce or eliminate the truth predicate in a "natural" deduction system satisfying presumptions $(\alpha)$ to $(\theta)$. It is designed so as not to validate the source of contradictions identified above.

(=E) $b=c, \varphi(b) \vdash \varphi(c)$, if $b$ does not occur in the scope of $\mathrm{T}$ in $\varphi$; and, if it does, $b=c$, $\vdash \varphi(b)$ entails $\vdash \varphi(c)$ only if $\varphi$ is Tor $\sim$ T.

The first clause permits substitution of identicals when $b=c$ for any occurrence of $b$ in $\varphi$ except $b$ in the scope of the truth predicate, whether or not $\varphi(b)$ is proven under an assumption and whether or not $\varphi(b)$ is compositionally complex. The last clause is for the case that $\varphi(b)$ is a theorem (i.e. derived from premises, rules and axioms; but not a step under any undischarged assumptions), and $\varphi$ is not some complex predicate but simply the truth predicate or its negation. In which case, given that the system satisfies presumption $(\theta),=\mathrm{E}$ cannot result in inconsistent overdetermination.

\subsection{Two Examples of Using These Rules}

Consider a chain of claims about a contingent matter.

(A) What Ann says is "Money grows in beehives."

(B) What Beth says is "What Ann says is not true."

(C) What Cat says is "What Beth says is not true."

One may reason systematically about these.

(4) Money does not grow in beehives Premise.

(5) "Money grows in beehives" is true iff money grows in beehives

CT-schema.

(6) "Money grows in beehives" is not true. (4), (5) Sentential Logic (SL)

$\begin{array}{ll}\text { (7) What Ann says is not true } & \text { (A), (6) }=\mathrm{E} \text {. }\end{array}$

(8) "What Ann says is not true" is true iff what Ann says is not true CT-schema.

(9) "What Ann says is not true" is true (7), (8) SL.

(10) What Beth says is true (9), (B) $=$ E.

(11) "What Beth says is not true" is true iff what Beth says is not true CT-schema.

(12) "What Beth says is not true" is not true (11), (10) SL.

(13) What Cat says is not true

(12) $(\mathrm{C})=\mathrm{E}$.

There was no restriction on use of Identity Elimination because in the first place what Ann says does not contain the T-predicate, and subsequent steps about what Beth and Cath say are neither compound sentences nor under any undischarged assumptions.

The matter would be quite different if Ann had said "What Cat says is not true". Then:

(14) "What Cat says is not true" is true iff what Cat says is not true. CT-schema.

(15) What Ann says is "What Cat says is not true" Premise. 
But one cannot validly derive $\left(16^{*}\right)$ below by $=\mathrm{E}$ from (14) and (15), because (14) is a compound expression. Nor can this be done by making some assumption and attempting to use $=\mathrm{E}$ under an assumption, because one would need to use $=\mathrm{E}$ to substitute within the scope of the truth predicate.

$\left(16^{*}\right)$ What Ann says is true iff what Cat says is not true.

\subsection{A crucial Objection and Response Regarding Use of the Diagonal Lemma}

Isn't diagonalization achievable using Gödel's Diagonal Lemma without using Identity Elimination? An instance of that lemma is $\lambda$ iff $\sim T\langle\lambda\rangle$, for some sentence $\lambda$. If this is valid, a contradiction will follow by Sentential Logic (SL) from the instance of the CT-schema for $\lambda$.

While diagonalization is also proven using Gödel's Diagonal Lemma, that lemma is actually proven using functions and substitution of identicals. Indeed, if the proof uses first-order logic with identity and functions, the proof of the relevant instance of the lemma does use Identity Elimination within the scope of the truth predicate. In a system with this rule restricted, this instance of the Lemma will not follow. To demonstrate this, I will provide some details. Consider the following proof of the lemma using the self-predication function, $s p$, as per Quine (1995).

The self-predication of an open sentence is identical with a canonical name for that open sentence predicated by the predicate of that open sentence. In general, $s p(\langle\varphi(x)\rangle)=\langle\varphi(\langle\varphi(x)\rangle)\rangle$.

$$
\begin{gathered}
\text { So that, } s p(\langle\varphi(s p(x))\rangle)=\langle\varphi(s p(\langle\varphi(s p(x))\rangle))\rangle \\
\text { and in particular, } s p(“ \sim \mathrm{T}(\operatorname{sp}(x)) ”)={ }^{\prime} \sim \mathrm{T}\left(\operatorname{sp}\left(\left(^{(} \sim \mathrm{T}(\operatorname{sp}(x))\right\rangle\right)\right)
\end{gathered}
$$

Abbreviating the left-hand side by " $a$ ", the above is of the same form as (1):

$$
a=\text { “ } \mathrm{T}(a) "
$$

Here, following Quine, is a proof of the Diagonal Lemma. (Of course, there is a lot more involved in Gödel's proof in addition to syntactic functions and substitution that concerns a theory of arithmetic.)

Diagonal lemma. Given canonical naming of a language's expressions and a syntactic function to the effect of self-predication, $s p$, then for any $\varphi(x)$, there is a closed formula $\psi$ such that:

$$
\psi \leftrightarrow \varphi(\langle\psi\rangle)
$$

Proof:

(17) $\varphi(s p(\langle\varphi(s p(x))\rangle)) \leftrightarrow \varphi(s p(\langle\varphi(s p(x))\rangle))$

SL

(18) $s p(\langle\varphi(s p(x))\rangle)=\langle\varphi(s p(\langle\varphi(s p(x))\rangle))\rangle$ Self-predication function

(19) $\varphi(s p(\langle\varphi(s p(x))\rangle)) \leftrightarrow \varphi(\langle\varphi(s p(\langle\varphi(s p(x))\rangle))\rangle)$

(17) $(18)=\mathrm{E}^{\star}$

(20) Let " $\psi$ " abbreviate " $\varphi(s p(\langle\varphi(s p(x))\rangle))$ " in (19).

The asterisk mark after "=E" flags our present concern about instances of that rule where $\varphi$ is a predicate that is or incorporates the truth predicate. Were that instance of that rule valid in our system, it would seem $\sim \mathrm{T}(\operatorname{sp}(“ \sim \mathrm{T}(\operatorname{sp}(x))$ ”)) 
$\sim \mathrm{T}\left(\right.$ (“ $\left.\sim \mathrm{T}\left(s p\left({ }^{\prime} \sim \mathrm{T}(s p(x))\right)\right)\right)$ "). Let “ $\lambda$ ” abbreviate the left hand-side of this biconditional, and one has $\lambda \leftrightarrow \sim \mathrm{T}(\lambda)$. But by the CT-schema $\mathrm{T}(\lambda) \leftrightarrow \lambda$, and we would have our contradiction. Alternatively, again abbreviate " $\operatorname{sp}\left({ }^{\prime} \sim \mathrm{T}(s p(x))\right.$ )" $)$ by $a$, and it seems the proof of the lemma yields $\sim \mathrm{Ta} \leftrightarrow \sim \mathrm{T}$ (“ $\sim \mathrm{Ta}$ "). But by the CT-schema $\mathrm{T}(“ \sim \mathrm{T} a$ ”) $\leftrightarrow \sim \mathrm{T}$ a; and again, our contradiction would follow. However, in each of these cases (19) would have to use Identity Elimination in the scope of the truth predicate, which is illicit in a system implementing our proposals.

\section{The Liar Hypodox}

The following is assertable in a system implementing our proposals, even if $a=$ $\langle\sim \mathrm{T} a\rangle$.

(21) $\mathrm{T}\langle\sim \mathrm{T} a\rangle$ iff $\sim \mathrm{Ta} \quad$ CT-schema

In classical logic so extended, so is this instance of the Law of Excluded Middle:

(22) $\mathrm{Ta} \vee \sim \mathrm{Ta}$

Furthermore, it follows classically that

(23) $(\sim \mathrm{T} a \& \mathrm{~T}\langle\sim \mathrm{T} a\rangle) \vee(\sim \sim \mathrm{T} a \& \sim \mathrm{T}\langle\sim \mathrm{T} a\rangle)$

No contradiction follows, because of the restriction on Identity Elimination. Like the Truth-teller, the semantic value of a Liar sentence is now underdetermined. One can prove it is true or not true (22), but no principle determines which. It is hypodoxical. As per (23), the Liar sentence is either the case but is true, or not the case and not true. There is then a residual question about the semantic value of each of the Liar and the Truth-teller sentences. The residual question could be answered in a number of ways without revising this solution that avoids Explosion.

\section{The Simple Liar}

In the above, I used the Strengthened Eubulidean Liar paradox. My derivations are comparable to presentations in Tarski (1944). This solution also applies straightforwardly to the so-called Simple Liar, concerning 'This sentence is false' rather than "This sentence is not true".

Consider the following, where " $P$ " abbreviates say "The Simple Eubulidean Liar". (This derivation can be compared to others in the literature, such as the derivation in Cook (2013).)

(24) $l=\langle\mathrm{F}\rangle \quad$ Premise,

(i.e. 'The Simple Eubulidean Liar is identical with "The Simple Eubulidean Liar is false"')

(25) $\mathrm{T}\langle\mathrm{F} /$ iff $\mathrm{F} l \quad$ CT-schema

(26) $\mathrm{T}$ liff $\mathrm{F}$ from (24) directly by naive $\mathrm{T}$-schema ${ }^{*}$, or from (24) and

(25) by $=\mathrm{E}^{\star}$.

(27) $\mathrm{T} l \& \mathrm{~F} l$

SL

Line (26) does not follow in our logic. The naive T-schema is restricted to the 
CT-schema, and, given our restriction on $=\mathrm{E},(26)$ does not follow by that rule. There is one minor complication of adding a falsity predicate, $\mathrm{F}$, to our language. So that it might seem one could derive

(28) $\mathrm{T}\langle\mathrm{F}\rangle$ iff $\mathrm{F}\langle\mathrm{F}\rangle-(24)$ and $(25)$ by $=\mathrm{E}$.

But introducing the truth and falsity predicates as unrelated predicates would court fallacies. We have enough paradoxes without inviting more. What I suggest is that the system be extended with a truth predicate, then a falsity predicate is added as an abbreviation. There are two candidates worth considering (given that the logic is using classical negation). If "is false" simply abbreviates " $\sim \mathrm{T}$ ", then our restriction on $=\mathrm{E}$ straightforwardly invalidates deriving (28) in this way. If the falsity predicate is such that " $\mathrm{F}\langle\ldots\rangle$ " in effect abbreviates " $\mathrm{T}\langle\sim \ldots\rangle$ ", then our restriction on $=\mathrm{E}$ also invalidates deriving (28) in this way.

There are of course many other approaches to the Liar. Remember that Identity Elimination (=E) is an implementation (so to speak) of substitution of identicals in first-order logic. The sentential logic that is used to derive (27) from (26) implements logical principles such as Bivalence and Non-contradiction. For discussion of the roles of these principles in deriving the Liar see (Cook 2013). It is interesting to note, as per Cook's discussion, that Bivalence is a principle relevant to the sentential logic of the Simple Liar in the way that the Law of Excluded Middle is a principle relevant to the sentential logic of the Strengthened Liar. But I have not discussed Sentential Logic at all! The Liar appears to me to be a predicate paradox, involving the truth predicate; therefore, it seems to me that remediation is to be done in predicate logic.

This accords with my restrictions on introducing and eliminating the truth predicate and the restriction on eliminating the identity relation (i.e. my restriction on the $=\mathrm{E}$ rule). My purpose in this article thus far has been to introduce this approach to the Eubulidean Liar in some detail, making clear the presumptions on which it relies; and to thus exemplify in some detail a new way of dealing with old paradoxes - defuse them to hypodoxes. The Liar Hypodox poses a residual issue about the Liar's semantics; but in defusing the Liar Paradox to the Liar Hypodox, quite enough has been done to avoid deriving a contradiction from the Eubulidean Liar.

Would that the Liar in general was quite as simple as the Eubulidean Liar. We will need one more restriction on universal quantifier elimination $(\forall \mathrm{E})$ to address the Epimenides paradox. This is clearly a variation of the Liar, or dare I say, one among a number of generalizations of the Liar.

\section{Generalizations of the Liar}

How generally does this approach defuse related paradoxes to hypodoxes? One may wonder about the class of paradoxes to which this solution applies, and in particular whether it applies to some that may be considered as generalizations of the Liar. I outline answers to these questions in this section.

Broadly construed, generalizations of the Liar include: truth-functional varia- 
tions; Curry's paradox, where it uses the truth predicate; the Epimenides, which involves quantification; and Yablo's paradox, which involves an infinite list of Liar-like statements. Moreover, the Unsatisfied paradox is a generalization of the Liar using a truth relation instead of the truth predicate (Eldridge-Smith 2015). In subsequent subsections, I will demonstrate how this solution applies to each of these.

I note first though that Eldridge-Smith (2015) distinguishes between his Unsatisfied paradox and a variation of Grelling's paradox as different kinds of paradoxes having different pathologies. (To the best of my knowledge, this variation of Grelling's paradox that explicitly uses a truth relation was first presented in Quine 1976. Not everyone accepts that it is a variation of Grelling's, but that is merely a dispute about how to best name this paradox.) The arguments for this distinction are in Eldridge-Smith (2015). In this article, I presume this distinction.

( $\iota$ The Liar is the same kind of paradox as the Unsatisfied paradox, and a distinct kind from Grelling's paradox, because they have different pathologies (as argued in Eldridge-Smith 2015).

In the proofs that follow, I enforce use of the CT-schema. Nevertheless, to exemplify how contradictions are avoided, I do represent the proofs using unrestricted $=\mathrm{E}$ but mark it with an asterisk where it violates its restrictions in our system. Those lines are therefore invalid in the system intended to implement our solution. The proofs that follow may require expertise with first-order logic and at one point even some familiarity with omega-inconsistency. The general reader may wish to browse the rest of this section and resume close reading in the next.

\subsection{Truth-Functional Variations of the Liar}

The same general strategy works for truth-functional variants of the Eubulidean Liar. Here is an example of a proof of the so-called ESP paradox (Eldridge-Smith 2012).
(29) $b=\langle\mathrm{T} b \equiv \mathrm{Q}\rangle$ Premise
(30) $\mathrm{T}\langle\mathrm{T} b \equiv \mathrm{Q}\rangle \leftrightarrow(\mathrm{T} b \equiv \mathrm{Q})$
CT-schema
(31) $\mathrm{T} b \leftrightarrow(\mathrm{T} b \equiv \mathrm{Q})$
(29), (30) $=\mathrm{E}^{*}$
(32) Q
by SL, presuming the main biconditional

in the CT-schema is also a material conditional.

The interesting feature of the ESP is that if $\mathrm{Q}$ is true, then $b$ is hypodoxical; but if $\mathrm{Q}$ is false, $b$ is paradoxical. $\mathrm{Q}$ could be any statement though.

The above proof interprets the biconditional in the CT-schema as a material conditional. This is not a presumption of our solution to the Liar; nevertheless, our solution can be implemented in an extension of first-order classical logic. The proof above is representative of other proofs of truth-functional variations of the Liar with respect to having to make an invalid use of $=\mathrm{E}$, given our restriction to the CT-schema. The details of the Sentential Logic at issue vary but are not relevant to our proposal. 


\subsection{Curry's Paradox Using a Truth Predicate}

Cook demonstrates that the Liar can be viewed as a special case of Curry's (Cook 2013: 76). Conversely, I consider that that Curry's paradox, when using the truth predicate, is one among a number of ways of generalizing the Liar. An interesting feature of Curry's paradox is its proof does not rely on use of negation. Our approach to the Liar did not affect negation anyway. Here is a proof, where Q is any sentence.
(33) $c=\langle\mathrm{T} c \rightarrow \mathrm{Q}\rangle$
premise
(34) $\mathrm{Tc}$ assumption
(35) $\mathrm{T}\langle\mathrm{T} c \rightarrow \mathrm{Q}\rangle$
(34), (33) $=\mathrm{E}^{\star}$
(36) $\mathrm{T} c \rightarrow \mathrm{Q}$
(35) $\mathrm{TE}$
(37) Q
(36) (34) $\rightarrow \mathrm{E}$
(38) $\mathrm{T} c \rightarrow \mathrm{Q}$
(34)-(37) $\rightarrow$ I [34]
(39) $\mathrm{T}\langle\mathrm{T} c \rightarrow \mathrm{Q}\rangle$
(38) TI
(40) $\mathrm{Tc}$
(39), (33) =E
(41) Q
(38), (40) $\rightarrow \mathrm{E}$

It is the use of $=\mathrm{E}$ to obtain line (35) that is invalid in our intended system. In this derivation it is used to substitute a coreferential term within the scope of the truth predicate under an undischarged assumption. Other derivations of Curry's may not use assumptions, as this proof does at line (34), but would invalidly use $=\mathrm{E}^{\star}$ to perform a similar substitution into a complex expression, given our restriction to the CT-schema. Still other derivations would use the Diagonal Lemma, but those instances of that lemma cannot be validly derived in our intended system for the same reasons demonstrated in Section 3.3.

\subsection{Restriction of Universal Quantifier Elimination: The Epimenides}

Epimenides the ancient Cretan philosopher, priest and poet once said in a poem long lost that Cretans always lie. For present purposes, lying is just saying something untrue, and all other Cretan statements are taken to be false. Actually, under a number of circumstances Epimenides' statement generates a number of paradoxical conundrums. For a brief philosophical history of variations of the Epimenides, see Eldridge-Smith (2004).

Let " $D$ " represent the predicate "is said by a Cretan", and consider the proof below.
(42) $\mathrm{D}\langle\forall \mathrm{x}(\mathrm{Dx} \supset \sim \mathrm{Tx})\rangle$
premise
(43) $\forall \mathrm{x}(\mathrm{Dx} \supset \sim \mathrm{Tx})$ assumption
(44) $\mathrm{D}\langle\forall \mathrm{x}(\mathrm{Dx} \supset \sim \mathrm{Tx})\rangle \supset \sim \mathrm{T}\langle\forall \mathrm{x}(\mathrm{Dx} \supset \sim \mathrm{Tx})\rangle(43) \forall \mathrm{E}^{\star} \mathrm{x} /\langle\forall \mathrm{x}(\mathrm{Dx} \supset \sim \mathrm{Tx})\rangle$
(45) $\sim \mathrm{T}\langle\forall \mathrm{x}(\mathrm{Dx} \supset \sim \mathrm{Tx})\rangle$
(42) (44) Modus Ponens (MP)
(46) $\mathrm{T}\langle\forall \mathrm{x}(\mathrm{Dx} \supset \sim \mathrm{Tx})\rangle$
(43) $\mathrm{TI}$
(47) $\sim \forall \mathrm{x}(\mathrm{Dx} \supset \sim \mathrm{Tx})$
(45) $(46) \sim \mathrm{I}[43]$
(48) $\exists \mathrm{x}(\mathrm{Dx} \& \mathrm{Tx})$
(44) Quantifier Negation (QN), SL

I pause here to remark, as Alonzo Church does, how surprising it is that 
merely from a Cretan saying that all Cretan statements are untrue that it seems to follow that there exists a true Cretan statement (Alonzo Church 1946)! I have marked $\forall E$ with an asterisk at line (44), which will be explained later in this section. Note also one can assert the falsity of Epimenides statement as follows.

(49) $\mathrm{T}\langle\sim \forall \mathrm{x}(\mathrm{Dx} \supset \sim \mathrm{Tx})\rangle$

(47) $\mathrm{TI}$

Given all other Ds, if any, are $\sim \mathrm{T}$ :

(50) $\forall \mathrm{x}((\mathrm{Dx} \& \mathrm{x} \neq\langle\forall \mathrm{x}(\mathrm{Dx} \supset \sim \mathrm{Tx})\rangle) \supset \sim \mathrm{Tx}) \quad$ Premise

(51) $\mathrm{Da} \& \mathrm{Ta}$ Assumption

(52) $(\mathrm{Da} \& \mathrm{a} \neq\langle\forall \mathrm{x}(\mathrm{Dx} \supset \sim \mathrm{Tx})\rangle) \supset \sim \mathrm{Ta}$

(50) $\forall \mathrm{E}$

(53) $\mathrm{a}=\langle\forall \mathrm{x}(\mathrm{Dx} \supset \sim \mathrm{Tx})\rangle$

(51) (52) SL

(54) $\mathrm{Ta}$

(51) \& E

(55) $\mathrm{T}\langle\forall \mathrm{x}(\mathrm{Dx} \supset \sim \mathrm{Tx})\rangle$

(54) $(53)=\mathrm{E}^{\star}$

(56) $\forall \mathrm{x}(\mathrm{Dx} \supset \sim \mathrm{Tx})$

(55) $\mathrm{TE}$

(57) $\forall \mathrm{x}(\mathrm{Dx} \supset \sim \mathrm{Tx})$

(48), (51)-(56) $\exists \mathrm{E}[51]$,

\# with line (47)

The inference of line (55) by $=\mathrm{E}$ is invalid. So line (57) does not follow, and therefore the contradiction between it and line (47) is avoided. However, without a further restriction, our theory is an omega-inconsistent theory; even though it is not syntactically inconsistent. For any particular Cretan statement, including Epimenides own, is not true as per lines (49) and (50). Nevertheless, we cannot prove-not by the above means anyway-that all Cretan statements are not true! If we could, the system would be inconsistent. The usual way of doing it would be as above, but the use of $=\mathrm{E}$ marked with an asterisk is invalid in our system. In other words, without further restriction, we can prove that some Cretan statement is true but there is no particular Cretan statement, in the given circumstances, that we can assert is true. Better minds than mine may be able to cope with omega inconsistency; perhaps an omega-paraconsistent theory would be interesting. Personally, I think the way forward involves finding one further fallacy. And I am not the first to think that there is one! As I already mentioned, Alonzo Church (1946) found the proof represented by lines (42) to (48) surprising, even paradoxical.

Seeking guidance again from considerations about related hypodoxes, there is a redundancy in proving related hypodoxes between use of $\forall \mathrm{E}$ and use of truth introduction (TI). Once again, those hypodoxes need not rely on TI; they can prove an instance of that rule using universal instantiation, which in first-order logic is the rule for universal quantifier elimination, $\forall \mathrm{E}$.

Consider the hypodox when instead Epimenides says 'All Cretan statements are true' and instead all other Cretan statements are true. What is the truth-value of this alternative Epimenides statement? Well, it could consistently be true or false, but nothing seems to determine which. It is hypodoxical. Compare the following proof to the first lines of our proof of the Epimenides above.
(58) $\mathrm{D}\langle\forall \mathrm{x}(\mathrm{Dx} \supset \mathrm{Tx})\rangle$
Premise
(59) $\forall \mathrm{x}(\mathrm{Dx} \supset \mathrm{Tx})$ Assumption
(60) $\mathrm{D}\langle\forall \mathrm{x}(\mathrm{Dx} \supset \mathrm{Tx})\rangle \supset \mathrm{T}\langle\forall \mathrm{x}(\mathrm{Dx} \supset \mathrm{Tx})\rangle$
(59) $\forall E^{*}$ 
(61) $\mathrm{T}\langle\forall \mathrm{x}(\mathrm{Dx} \supset \mathrm{Tx})\rangle$

(62) $\forall \mathrm{x}(\mathrm{Dx} \supset \mathrm{Tx}) \supset \mathrm{T}\langle\forall \mathrm{x}(\mathrm{Dx} \supset \mathrm{Tx})\rangle$

(58) (60) MP; or (59) TI

(59)-(58) つ I [59]

In the $4^{\text {th }}$ line of this proof we have derived a truth (under an assumption). It could be derived in two ways, either by using $\forall \mathrm{E}$ (and MP), or by using TI. But the correlative results of using $\forall \mathrm{E}$ (and MP) in proving line (45) of the Epimenides was the contradiction of what results from using TI in that proof. Motivated by this, either $\forall \mathrm{E}, \mathrm{MP}$ or TI should be restricted to remedy this source of omega-contradiction. I think this is a predicate paradox, requiring a predicate remedy; so, I do not think MP is the cause of this inconsistency. In other words, I can see no reasonable way of restricting MP that avoids this inconsistency without making many more seemingly valid inferences illicit. And, again, TI is the rule intended to introduce truth, not $\forall \mathrm{E}$. Therefore, I propose the following restriction on $\forall \mathrm{E}$.

$(\forall \mathrm{E}) \forall \mathrm{x}(\varphi(\mathrm{x})) \vdash \varphi(b)$ where $b$ replaces all and any $\mathrm{x}$ in $\varphi$ if $b$ does not occur in the scope of $\mathrm{T}$ in $\varphi$; and, if it does, $\vdash \forall \mathrm{x}(\varphi(\mathrm{x}))$ entails $\vdash \varphi(b)$ only if $\varphi$ is Tor $\sim \mathrm{T}$.

The first clause permits instantiation for all occurrences of $\mathrm{x}$ in $\varphi$ except in the scope of the truth predicate, whether or not $\forall \mathrm{x}(\varphi(\mathrm{x}))$ is proven under an assumption and whether or not $\forall \mathrm{x}(\varphi(\mathrm{x}))$ is a compositionally complex. The last clause is for the case that $\forall \mathrm{x}(\varphi(\mathrm{x}))$ is a theorem (i.e. derived from premises, rules and axioms; but not a step under any undischarged assumptions), and $\varphi$ is not some complex predicate but simply the truth predicate or its negation. In which case, given that the system satisfies presumption $(\theta), \forall \mathrm{E}$ cannot result in inconsistent overdetermination.

\subsection{Yablo's Paradox}

Whereas in one form of the Epimenides, all other Cretans' statements are false, Yablo's paradox references an infinite list of statements each of which says all the subsequent statements in the list are not true (Yablo 1993). Thus, where "Y" abbreviates "the ith Yablo statement", $\mathrm{Y}_{\mathrm{i}},=\left\langle\forall \mathrm{x}\left(\mathrm{x}>i \supset \sim \mathrm{TY}_{\mathrm{x}}\right)\right\rangle$. The interesting feature about Yablo's paradox is that it prima facie does not rely on self-reference, or even circular reference. Given our restriction to the CT-schema, here is part of a proof of Yablo's paradox:

(63) $\mathrm{Y}_{1}=\left\langle\forall \mathrm{x}\left(\mathrm{x}>1 \supset \sim \mathrm{TY}_{\mathrm{x}}\right)\right\rangle$

premise

(64) $\forall \mathrm{x}\left(\mathrm{x}>1 \supset \sim \mathrm{TY}_{\mathrm{x}}\right)$ assumption

(65) $2>1 \supset \sim \mathrm{TY}_{2}$ (64) $\forall E^{*} x / 2$

(66) $\sim \mathrm{TY}_{2}$ $2>1,(65) \mathrm{SL}$

(67) $\forall \mathrm{x}\left(\mathrm{x}>2 \supset \sim \mathrm{TY}_{\mathrm{x}}\right) \rightarrow \mathrm{T}\left\langle\forall \mathrm{x}\left(\mathrm{x}>2 \supset \sim \mathrm{TY}_{\mathrm{x}}\right)\right\rangle$ CT-schema, SL

(68) $\sim \forall \mathrm{x}\left(\mathrm{x}>2 \supset \sim \mathrm{TY}_{\mathrm{x}}\right)$ (66) $=\mathrm{E}^{\star},(67) \mathrm{SL}$

(69) $\sim \forall \mathrm{x}\left(\mathrm{x}>1 \supset \sim \mathrm{TY}_{\mathrm{x}}\right)$

(64) $-(68) \sim \mathrm{I}[64]$

(70) $\exists \mathrm{x}\left(\mathrm{x}>1 \& \mathrm{TY}_{\mathrm{x}}\right)$

(69) QN, SL

One can see that this particular derivation is invalid in our intended system at two steps, line (65) and line (68). For present purposes, we need not continue with this derivation. Yablo's paradox is at the centre of a debate about whether it is a relative of the Liar that does not actually use circular reference. Nothing 
among our present proposals depends on this; although it is a crucial issue for some other proposed approaches to the Liar and its relatives.

\subsection{Grelling's Is a Different Kind of Paradox}

Corresponding to the CT-schema is a Canonical Truth Relation schema

CTR-schema: $\langle\varphi(\mathrm{x})\rangle$ is true of $b \leftrightarrow \varphi(b)$

For example,

"Ariadne loves $\mathrm{x}$ " is true of Theseus $\leftrightarrow$ Ariadne loves Theseus.

Now, following Quine (1976), using the enquotation of “ $x$ is true of itself” for both the name of an open sentence and as a term, we have the following instance of this schema:

(71) " $x$ is not true of itself" is true of " $x$ is not true of itself" iff " $x$ is not true of itself" is not true of itself

which is grammatically equivalent to:

(72) " $x$ is not true of itself" is true of itself iff " $x$ is not true of itself" is not true of itself

Such is Quine's variation of Grelling's paradox. Quine himself claims this is Grelling's paradox. Eldridge-Smith (2015) distinguishes it as a different kind of paradox from those depending on substitution of identicals and schemata such as the CT-schema and CTR-schema that are restricted to canonical names. That this distinction is correct is my presumption $(\iota)$ above. The paradox using the truth relation that is a generalization of the Liar is Eldridge-Smith's (2012b; 2015) Unsatisfied paradox.

\subsection{The Unsatisfied Paradox}

The Unsatisfied paradox is so named after the satisfaction relation, the converse of the truth relation. Here, using the truth relation, is a variation of Eldridge-Smith's Unsatisfied paradox (Eldridge-Smith 2015: p. 88), the so-called Untrue paradox.

(1) $s=\langle s$ is not true of $\mathrm{x}\rangle$ Premise

(2) $\langle s$ is not true of $\mathrm{x}\rangle$ is true of $t$ iff $s$ is not true of $t$

CTR-schema

(3) $s$ is true of $t$ iff $s$ is not true of $t$ (1), (2) $=\mathrm{E}^{*}$

An interesting feature of the Unsatisfied paradox (and its Untrue variation) is that $t$ could be anything: the Eiffel tower, Chicago, Edith Piaf, Hypatia or snow. But our present concern is that (3) would be invalid in our system, given a natural generalization of our restrictions on $=\mathrm{E}$ from the truth predicate extended to the truth relation.

\section{Consistency}

In general, if a logic with identity is consistent and satisfies presumptions $(\alpha)$ through $(\eta)$, it can be extended with a truth predicate in this way. In the extension, since truth can only be introduced or eliminated where it predicates a canonical name, this would force the use of Identity Elimination or universal instantiation to derive a Liar paradox, but that usage is restricted. Specifically, an 
underlying classical first-order logic with identity is consistent. I presume its extension with the CT-schema but unrestricted Identity Elimination satisfies $(\theta)$. Then, the resultant extension of that system with a truth predicate implementing the three proposed restrictions is consistent, or at least free of contradictions due to using an informal T-schema and having more than one way of introducing and eliminating the truth predicate.

This systematic extension of a first-order system with a truth predicate with these restricted principles does not require any modifications to the theorems of the underlying logic it extends-certainly with respect to extending classical first-order logic with identity. Not a single theorem of that underlying logic is revoked by this extension, even though the application of Identity Elimination and universal instantiation are restricted. These restrictions affect only new theorems of the extended system.

There is still much work to do. If the system is further extended with a truth relation, relatives of the Liar, such as the unsatisfied paradox can be defused to hypodox by an extension of our restrictions to the truth relation. However, as per Section 6.5 and given presumption $(\iota)$, there is another kind of paradox of the truth relation. I do have other proposals to defuse that other kind of paradox, but this article is concerned with the Liar and its ilk.

\section{Conclusion}

Defusing the Liar paradox to the Liar hypodox demonstrates in detail how a paradox can be defused to a hypodox. $A$ fortiori, this demonstrates a new way of dealing with paradoxes: defuse them to hypodoxes. This must be done methodically. My method was to identify three sources of contradiction and invalidate those sources of contradiction by making some minimal restriction. While doing so, I have been careful to state my presumptions.

I have presented each source of contradiction and my recommended restriction in a certain sequence. This sequence reflects my confidence in each. I am convinced, given my stated presumptions, that the informal T-schema is fallacious as I argued in Section 2. I hope that this result will be of general use in research towards a theory of truth. To avoid this source of contradiction, I recommend restricting rules for truth introduction and elimination to canonical names (and the derived CT-schema). This restriction seems a reasonable restriction to avoid this source of contradiction since the contradiction arises from the informality of the T-schema, and the CT-schema validates all but the informal instances of the $\mathrm{T}$-schema. As the $\mathrm{T}$-schema has intuitive support and the CT-schema only validates a proper subset of its instances, the CT-schema should also have intuitive support.

More is clearly required because the CT-schema together with unrestricted substitution of identicals would entail all instances of the original T-schema, another source of contradiction. (Substitution of identicals is implemented by the Identity Elimination rule in first-order logic.) Truth Introduction and Elimina- 
tion are the intended rules for introducing and eliminating the truth predicate. Yet in Section 3, I find that for the hypodoxical Truth-teller, =E can produce the same result as these rules of truth; but in the corresponding circumstances for the Liar, $=\mathrm{E}$ results in contradicting what is derived by TI and TE.

In remediating this source of contradiction, I think my argument that Identity Elimination should be restricted is reasonable. One could instead try to further restrict Truth Introduction and Truth Elimination. There are many ways in which that might be done (Friedman \& Sheard 1987). Generally, further restricting those rules of truth leads to weaker logics. My reasoning for preferring to restrict Identity Elimination is that that rule is not actually true to name, whereas Truth Introduction and Elimination are, and should remain the rules for introducing and eliminating the truth predicate. For example, in the inference from $a=b, \mathrm{~T}\langle\mathrm{P} a\rangle$ to $\mathrm{P} b$, no occurrence of "=" has actually been eliminated from " $\mathrm{T}\langle\mathrm{P} a\rangle$ "; whereas in the inference from $\mathrm{T}\langle\mathrm{P} a\rangle$ to $\mathrm{P} a$ by $\mathrm{TE}$, the truth predicate has clearly been eliminated.

For similar reasons in Section 6.3, I argued that a similar restriction is required on Universal instantiation in the extended logic. (Universal instantiation is implemented as the rule for universal quantifier elimination $(\forall E)$ in a first-order logic.) Again, I compared inferences made using $\forall E$ and Truth Introduction in a Truth-teller argument with the corresponding inferences made in the Epimenides argument, and argued that it is $\forall \mathrm{E}$ that needs to be restricted as it is a source of contradiction in these cases.

It is clear that my proposals make no modification to the theorems of first-order logic with identity. These can still be proven without using the truth predicate, and therefore none of my proposed restrictions will affect those proofs. Moreover, my restriction on theorems of the logic extended with a truth predicate is relatively minimalistic. It seems appropriate to me that all restrictions to defuse the Liar paradox are restrictions on predicate and quantifier rules in the extended logic, since I believe the Liar paradox is a predicate paradox.

To cap off my list of presumptions with a $10^{\text {th }}$, I have withheld formally declaring one last presumption till the end.

( $\kappa$ ) The Liar paradox has a complex cause.

I have been operating under this presumption throughout. I think that is why the Liar is so resistant to remediation. Since no single source of contradiction and remediation will solve the Liar paradox, given $(\kappa)$. Under this and my other presumptions, I have identified three sources of contradiction, and invalidated those sources of contradiction by making minimal restrictions. The result is that the Liar paradox is defused to a hypodox. Explosion does not follow from the Liar hypodox just as it does not follow from the Truth-teller.

Having said that, it is not proven that three sources of contradiction are all there are. Nevertheless, it is demonstrated that the Eubulidean Liar, in its Simple and Strengthened versions are reduced to hypodoxes. Then it is demonstrated that a variety of generalizations of the Liar no longer result in contradictions, viz. 
truth-functional variations of the Liar, Curry's paradox using the truth predicate, the classical Epimenides, Yablo's paradox and Eldridge-Smith's Unsatisfied paradox. Still, my consistency claim is a relative and conditional one.

In considering this, the reader may also wish to consider that defusing the Liar to a hypodox leaves open the question of how hypodoxes are to be addressed. Various semantic or epistemic solutions to hypodoxes may be compatible with the way in which we have defused the Liar.

\section{Acknowledgements}

A short version of this article was presented at the World Conference of Philosophy 2018 in Beijing at a Roundtable on Semantic Paradoxes chaired by Professor Chen Bo of Peking University. I am grateful for the discussion with members of that roundtable and the audience. A section of a paper that I presented at Universal Logic 2018 in Vichy in a session facilitated by Professor Franca D'Agostini and Professor Elena Ficara also considered reducing the Liar paradox to a hypodox. I am grateful for the discussion and feedback of those involved in the session and the audience.

\section{Conflicts of Interest}

The author declares no conflicts of interest regarding the publication of this paper.

\section{References}

Church, A. (1946). Review of "The Liar" by Alexandre Koyre. Journal of Symbolic Logic, 11, 131. https://doi.org/10.2307/2268325

Clark, M. (2012). Paradoxes from A to $Z$ (3rd ed.). Abingdon-on-Thames: Routledge. https://doi.org/10.4324/9780203096413

Cook, R. T. (2012). The T-Schema Is Not a Logical Truth. Analysis, 72, 231-239. https://doi.org/10.1093/analys/ans031

Cook, R. T. (2013). Paradoxes. Hoboken, NJ: John Wiley \& Sons.

Eldridge-Smith, P. (2004). The Cretan Liar Paradox. In J. Lloyd-Jones, P. Dobrez, \& L. Dobrez (Eds.), An ABC of Lying: Taking Stock in Interesting Times (pp. 72-92). Melbourne: Australian Scholarly.

Eldridge-Smith, P. (2007). Paradoxes and Hypodoxes of Time Travel. In J. Lloyd-Jones, P. Campbell, \& P. Wylie (Eds.), Art and Time (pp. 172-89). Melbourne: Australian Scholarly. https://philpapers.org/archive/ELDPAH.pdf

Eldridge-Smith, P. (2012). A Hypodox! A Hypodox! A Disingenuous Hypodox! The Reasoner, 6, 118-119.

Eldridge-Smith, P. (2012b). The Unsatisfied Paradox. The Reasoner, 6, 184-185.

Eldridge-Smith, P. (2015). Two Paradoxes of Satisfaction. Mind, 124, 85-119. https://doi.org/10.1093/mind/fzu183

Eldridge-Smith, P. (2018). Pinocchio against the Semantic Hierarchies. Philosophia, 46, 817-830. https://doi.org/10.1007/s11406-018-9948-y

Friedman, H., \& Sheard, M. (1987) An Axiomatic Approach to Self-Referential Truth. 
Annals of Pure and Applied Logic, 33, 1-21. https://doi.org/10.1007/s11406-018-9948-y

Halbach, V. (2014). Axiomatic Theories of Truth. Cambridge: Cambridge University Press. https://doi.org/10.1017/CBO9781139696586

Kripke, S. (1975). Outline of a Theory of Truth. Journal of Philosophy, 72, 690-716. https://doi.org/10.2307/2024634

Priest, G. (1979). The Logic of Paradox. Journal of Philosophical Logic, 8, 219-241. https://doi.org/10.1007/BF00258428

Quine, W. V. O. (1976). The Ways of Paradox. In His the Ways of Paradox and Other Essays (2nd ed., pp. 1-18). Cambridge, MA: Harvard University Press.

Quine, W. V. O. (1995). Truth, Paradox and Gödel's Theorem. In His Selected Logic Papers (Enlarged ed., pp. 236-241). Cambridge, MA: Harvard University Press.

Ripley, D. (2013). Revising up: Strengthening Classical Logic in the Face of Paradox. Philosophers' Imprint, 13, 1-13.

Sainsbury, R. M. (2009). Paradoxes (3rd ed.). Cambridge: Cambridge University Press. https://doi.org/10.1017/CBO9780511812576

Skyrms, B. (1970). Return of the Liar: Three-Valued Logic and the Concept of Truth. American Philosophical Quarterly, 7, 153-161.

Tarski, A. (1935). Der Wahrheitsbegriff in den formalisierten Sprachen. Studia Philosophica, 1, 261-405.

Tarski, A. (1944). The Semantic Conception of Truth and the Foundations of Semantics. Philosophy and Phenomenological Research, 4, 341-376. https://doi.org/10.2307/2102968

Yablo, S. (1993). Paradox without Self-Reference. Analysis, 53, 251-252. https://doi.org/10.1093/analys/53.4.251 